\title{
The NASA Juncture Flow Test as a Model for Effective CFD/Experimental Collaboration
}

\author{
Christopher L. Rumsey* \\ NASA Langley Research Center, Hampton, VA 23681
}

\begin{abstract}
The NASA Juncture Flow test, whose purpose is CFD validation for wing juncture trailing edge separation and progression, was designed from the outset to be a highly collaborative effort between CFD computationalists and experimentalists. This paper highlights key aspects of the planning and execution of the project, which has recently completed its first phase of wind tunnel testing. The joint CFD/experimental team is described, and its accomplishments to date are summarized.
\end{abstract}

\section{Introduction}

The NASA Juncture Flow (JF) experiment is a series of wind tunnel tests conducted in the NASA Langley 14by 22-Foot Subsonic Tunnel (14x22), taking place between November 2017 and March 2018, with follow-up testing scheduled in future years. The purpose of the experiment is to collect data to help assess/improve the ability of existing CFD models to predict the onset and extent of the three-dimensionally separated flow near the wing juncture trailing edge region of a full-span wing-body configuration. As will be described in the History section, CFD has typically proven to be unreliable for predicting this type of flow feature.

The flow physics of wing-body juncture flows is quite complex because it not only involves the interaction of two boundary layers in pressure gradient, but several distinct vortical structures can also be present [1]. Typically a large, unsteady horseshoe vortex as well as a smaller corner vortex are generated near the wing leading edge at the wing-body intersection, and wrap around the wing. There may also be a smaller corner vortex initiated by gradients of the Reynolds stresses [2]. All of these vortices pass downstream, influencing the flow field in the juncture region. Although there is general awareness that the presence of a larger horseshoe vortex can help suppress corner separation [3], at the current time the precise manner in which the vortical structures and associated Reynolds stresses interact to cause and/or suppress corner separation is not fully understood.

Juncture flows have been the subject of many previous studies. For example, Simpson and co-workers extensively investigated corner flow behavior of wings mounted on flat plates. However, that body of work did not include much focus on trailing-edge corner separation. Instead, the focus was more on the leading edge region and the horseshoe vortex behavior. The summary paper of Simpson [4] provides an overview of this and other similar work, with many relevant references provided. More recently, Gand et al. [5] and Bordji et al. [6] have reported results from experiment and CFD studies with corner separation. Their work also involved flow over a wing mounted on a flat plate.

The current JF experiment includes many new capabilities. It is a full-span wing-body configuration, and the corner flowfield region is measured using an internally-mounted laser-doppler velocimetry (LDV) system that makes use of windows in the model fuselage. This system allows for detailed flowfield measurements closer to walls near the corner region than is typically possible with traditional laser-based measurement systems (which can be limited by flare near surfaces). Many details about the lead-up to the current JF experimental/CFD campaign can be found in previous papers [7-12].

The primary intended purpose of the experiment is for CFD model validation $[13,14]$. As such, the main goal is "quantitatively determining the ability of the mathematical model (and its embodiment in a computer code) to predict the physical processes occurring in the model validation experiment" (quote, including italics, from the original reference [13]). To achieve this goal, effort is required to document a significant amount of information about the experimental facility, analog instrumentation and signal processing, boundary and initial conditions, fluid and material properties, test conditions, and system response quantities. As described in Aeschliman and Oberkampf [14], such an

\footnotetext{
${ }^{*}$ Research Scientist, Computational AeroSciences Branch, Mail Stop 128, Fellow AIAA.
} 
effort requires teamwork and cooperation between computationalists and experimentalists throughout the validation process.

This paper is a summary of the creation and workings of the joint experimental/CFD JF team. The author, who serves as the technical leader of the team, makes an effort here to provide an honest accounting of the strengths and weaknesses of the collaborative effort so that future teams may learn from it. The paper is organized as follows. First, a brief history of the project is outlined, then the JF team and its accomplishments to date are described in some detail. Finally, a summary of lessons learned is provided.

\section{History}

In past years, there has been significant confusion surrounding the computation of separated juncture flows. In the third Drag Prediction Workshop (DPW-3) [15], which used the DLR-F6 wing-body geometry, participants predicted a very wide range of side-of-body separation sizes with CFD. The same issue also came up for the Common Research Model (CRM) configuration at certain higher-than-cruise-angle-of-attack conditions in DPW-4 and DPW-5 [16]. Many subsequent studies looked at the issue, e.g., Refs. [17-19]. The computed juncture separated region appears to be influenced by a variety of factors other than just the turbulence model, including grid size, grid topology, and numerical treatments.

Over time, the CFD community has come to the consensus that linear (Boussinesq) turbulence models will generally produce unrealistic juncture separation results when using finer grids along with accurate numerics. In other words, when sufficiently reducing numerical discretization errors, a linear turbulence model will predict juncture separation inaccurately because of the construction of the turbulence model itself. Yamamoto et al. [20] first demonstrated that the use of a nonlinear quadratic constitutive relation (QCR) [21] could improve corner flow CFD predictions for the CRM, and Dandois [22] demonstrated improved predictions with QCR for the DLR-F6 wing-body configuration. Similarly, Rumsey [23] showed that nonlinear models were qualitatively better than linear models for the CRM, in terms of predicting expected separation size. Bordji et al. [6] came to similar conclusions for the Gand et al. [5] experiments. However, because of limited experimental validation data, determining the reasons why one model performs better than another is difficult.

The idea for the NASA Juncture Flow experiment was originally conceived by a few members of the DPW [15] steering committee. The experiment is intended to be primarily for the purpose of CFD validation for wing-juncture trailing edge separation onset and progression. A full-span semi-realistic wing-body configuration was envisioned, with flowfield measurements taken using an internally-mounted LDV system that makes use of windows in the model fuselage. This system allows for detailed flowfield measurements, including velocities and Reynolds stresses, very close to walls near the corner region that are difficult to measure any other way.

Although preliminary preparation work took place earlier, the JF team was "formally" formed and a leader assigned in summer 2014. Because of the importance of making the experiment useful for CFD validation, the project team was conceived from the beginning as a joint experimental and CFD effort. And even though the main emphasis of the project was on acquiring experimental data, the team's principal investigator (PI) was chosen to be a CFD practitioner to ensure strong representation from the computational side of the house throughout the lifetime of the project. As described in more detail in the next section, the team was assembled to include both experimental and CFD experts. Initial team work (summer 2014 through spring 2015) involved a significant amount of computational investigation. Attempts were made to use CFD to design the experiment. The original goal was to design a nonlifting wing/body configuration that was symmetric both top-bottom and left-right. The configuration was to be designed for transonic flow, with the freestream Mach number to be used to achieve a range of attached flow through separated flow. Ultimately, this initial CFD design effort was unsuccessful.

Around this time, many of the original requirements were changed for various reasons. Due to less availability of transonic wind tunnels of sufficient size (the internal LDV system housed within the model required a fuselage size of around 18 inches wide by 24 inches high, and the wing span was envisioned to be near 130 inches), the team decided to change the focus to subsonic flow testing. The $14 \times 22$ wind tunnel was selected for the experimental campaign. At the same time, use of Mach number to vary the separation extent was abandoned in favor of use of angle of attack, and the requirement for top-bottom symmetry was relaxed because the desired flow behavior could not be easily attained with those constraints.

Because of high uncertainties associated with designing a configuration to achieve the desired juncture flow behavior with CFD tools that may be inaccurate, a cautious approach was adopted for this project. Several preliminary "risk reduction" experiments were conducted during 2015 and 2016 in different wind tunnels using a variety of wing configurations, as described in Rumsey et al. [7] and Kegerise and Neuhart [8]. The risk reduction experiments allowed 


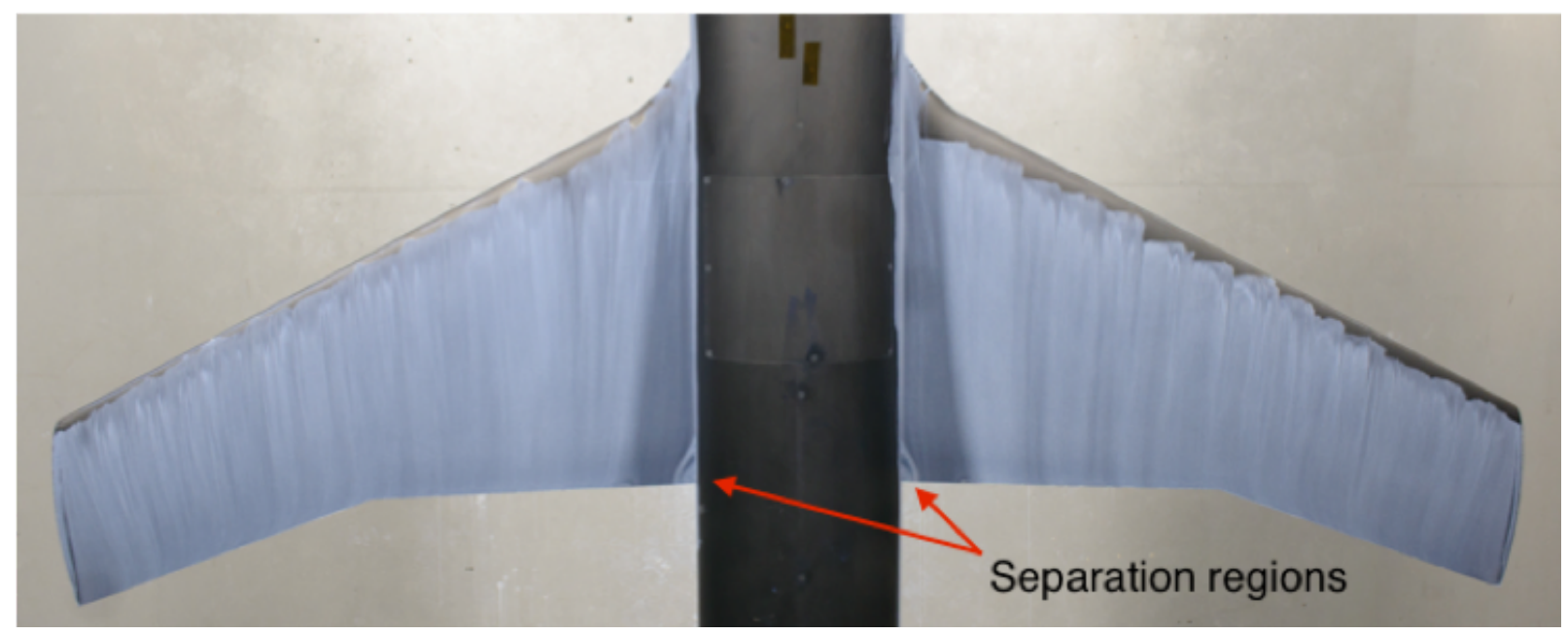

Figure 1. Example photograph showing separated regions at the wing root trailing edge of the JF model.

the team to more confidently select wing configurations that provide the desired flowfield characteristics; they also helped to determine the appropriate placement of instrumentation for the final experiment. An example of juncture flow separation from one of the risk-reduction experiments, visualized using oil flow, is shown in Fig. 1.

In the end, two different wing shapes were built. One was based on the DLR-F6 wing, and the other had an NACA 0015 shape at the root. The former achieved larger separation sizes, while the latter achieved fully-attached flow and incipient separation (no one wing tested could achieve both desired characteristics over the angle of attack range specified). Two fuselage noses were also built (one longer by 24 inches), to allow for the possibility of testing this parametric variation in the oncoming boundary layer thickness. Furthermore, both sets of wings were designed so that they could be installed either with or without a horn (fillet near the wing leading edge root). The presence of the horn influences the location and strength of the horseshoe vortex, which in turn affects the separation region.

During 2016 and 2017, some testing was also conducted in an effort to characterize the inflow freestream in the 14x22 wind tunnel using both Particle Image Velocimetry (PIV) and the Boeing Quantitative Wake-Survey System (QWSS) [24,25] MK-17 device. Although analysis of this work is still ongoing, some preliminary results were provided in Rumsey et al. [11].

\section{JF Team}

In this section, the team is described both in terms of its structure, as well as in terms of how it has functioned and what it has accomplished.

\section{A. Organization and Structure}

The JF team is comprised of a core group of consistent members, along with other auxiliary members who have participated only during team telecons or as needed. The JF team was formally begun in July 2014. At the start, there were approximately 25 total members. Of these, approximately 9 were CFD practitioners, 9 were experimentalists, 2 were model designers, 4 were managers, and 1 was an Uncertainty Quantification (UQ) consultant. The make-up was primarily NASA Langley Research Center personnel, along with 2 from NASA Ames, 4 from Boeing, 3 from private companies, 2 from universities, and 2 students. The team membership was somewhat fluid over the ensuing years, with some members dropping and others adding as various phases of the project unfolded. At the present time, there are still approximately 25 members with somewhat similar overall make-up as in the beginning. Only four or five team members work on this project as a significant fraction of their job over the entire length of the project.

As mentioned earlier, the author (a CFD practitioner) serves as the team leader. Three other CFD experts serve as part of the core group, while other computationalists were mostly involved only during the early design stages of the effort, then later became less involved. Approximately four experimentalists make up the core group, with others more involved during the testing phases of the project. The managers who attend the team meetings are mostly involved to monitor progress. Two of them are managers from the NASA Transformational Tools and Technologies (TTT) project 
that provided funding to the team, while the others are personnel managers.

From the beginning, because of the emphasis to ensure a focus on the CFD validation aspect of this experiment, a UQ consultant was brought onto the team. The impact of this decision will be discussed below. On the experimental side, two members of the company that developed the LDV system specifically designed for the needs of this test were also involved throughout the project.

Team meetings (by WebEx ${ }^{\mathrm{TM}}$ ) were held for one hour approximately every two weeks. At the TTT project level, progress was marked by occasional milestones. Also, special sessions were held at AIAA meetings, for which invited papers were written summarizing the project's status. Funding for the JF work was primarily provided by the TTT project, although a few other projects contributed funding for various parts of the work that were of interest to them. The majority of the funding was for the building of the JF model(s) and related hardware, instrumentation, and testing costs.

\section{B. Team Functioning and Dynamics}

A key feature of the JF team is that it has been comprised of both CFD and experimental experts from the very start. In terms of functionality, having both together was highly beneficial, as consultation and cross-fertilization was rapid. In terms of team dynamics, this make-up provided both advantages as well as challenges. Traditionally, CFD and experiment operate separately to a significant degree. Although each may consult the other during the course of a project, typically there is not an extremely tight linkage. As a result, all aspects from the two sides are not commonly considered when making decisions. The main advantage of a close coupling of the experiment and CFD on the same team is that that both experimental and CFD points of view are heard as a part of every team decision and in every step of the process. On the other hand, the close linkage at times can yield some tensions. The CFD and experimental cultures are somewhat different, and this difference can lead to clashes and frustrations. The author's main takeaway from this aspect of the team's experience is that, contrary to the popular aphorism, familiarity usually breeds trust and, if not agreement, at least a workable level of acceptance. The more that individuals from different backgrounds work closely together, the more they come to understand and accept each others positions, strengths, and weaknesses.

For the JF project, some of the aspects of the experiment considered important from the CFD perspective may not have been done, or perhaps would have been given a lower priority, if such a close working relationship had not been present between the experimentalists and the computationalists. Similarly, needs and priorities of the experimentalists would likely have been overlooked if CFD requirements and desires had been simply "thrown over the fence." In the very beginning of the team meetings, an effort was made to put in writing the goals and priorities for the experiment, taking into account both CFD and experimental perspectives. This goals document (provided in the Appendix) included a prioritization of how turbulence models would be assessed as well as a prioritized list of the data needed to accomplish this. This latter list included not only specified flowfield data, but also geometry and boundary condition information. The number one priority item (even above the data itself) was documentation of the test article geometry and surface, as fabricated, as assembled, and as tested in the wind tunnel. The goals document also emphasized the importance of including uncertainty measurements and analysis. It should be stressed that achieving all of the goals laid out in the document may not have ultimately been easy or even possible, but having them clearly written down helped to unify the team and aided with decision making.

Having team members from two different backgrounds (experiment and CFD) is one thing. Including team members from completely outside of the home organization is another. On the JF team, for example, there are a number of external members who do not participate consistently. Because they are remote, and not physically present, it is difficult for them to feel fully accepted to be a part of the team. It is possible that given more time and effort dedicated to team building, a more cohesive team spirit could have been engendered. However, such team building would have been very difficult to accomplish because of the team's non-locality, shifting membership, and other barriers. Nonetheless, the team continued to purposefully include the external members in every bi-weekly team meeting. The inclusion of these members added considerable benefit (in the author's opinion), as they often represented a different perspective when problems were encountered and difficult decisions needed to be made. It should be emphasized that the team strove to give due consideration to any team members' ideas before making a decision. The main takeaway here is that regular inclusion of many voices does not necessarily engender team cohesion, but it does add fresh perspectives that ultimately can help the team's final product.

A UQ consultant was included on the JF team near the start of the project because one of the team's sponsors had previously found the idea to be beneficial for a different project [26]. During the JF team meetings, the consultant's voice, which represented both an outsider to the home organization as well as an outsider in terms of his primary area of focus, was commonly the one that raised the most questions and posed the most alternative/unconventional ideas. 
Although sometimes frustrating for the team, these questions and ideas engendered more thought and debate than may otherwise have occurred.

\section{Accomplishments to Date}

As of the time of this writing, approximately 14 total weeks of testing on the JF model have taken place in two tunnel entries (this does not include the earlier risk-reduction experiments or wind tunnel inflow characterization experiments). By any standards, the JF project has been highly successful so far. Not only has high-quality flowfield and surface data been acquired toward the goal of CFD validation of juncture flow, this JF test also represents a breakthrough in its use of an on-board laser measurement system in a major NASA production wind tunnel. Considering the prioritized list of input data in the Goals Document included in the Appendix, most have been achieved or are on their way to being achieved.

1. To help ascertain the as-built and as-tested shape, the model with F6 wings was laser-scanned twice while in the tunnel (wind off), once with horns and once without. Photogrammetry was also used (wind on) to determine the wing deformation and twist under load.

2. For tunnel boundary conditions, test section wall pressures were recorded along the side walls and ceiling. Floor pressures were recorded in the diffuser using an additional pressure rail installed specially for this test. Wall rakes on the side walls and ceiling were used to record tunnel wall boundary layer thickness and growth. Attempts were made to measure details of the incoming tunnel freestream. Regarding the model itself, flowfield details were measured on the nose well upstream of the wing, helping to establish an on-board upstream boundary condition.

3. LDV was used to measure mean velocity components in three areas over the JF model: upstream on the nose, near the wing leading edge, and near the back of the wing upstream and within the separation region. The LDV probes provided simultaneous measurements of three velocity components. The measurement volume could be placed $200 \mu \mathrm{m}$ off the surface of windows and $500 \mu \mathrm{m}$ off the surface of the wing. Some complementary PIV data was also taken.

4. The LDV data acquired in (3) above is also being used to obtain second moments at the same locations.

5. Time-averaged steady surface pressures were obtained on the fuselage and both wings.

6. Boundary layer trips were used on the fuselage nose and on the wing upper and lower surfaces. Details regarding their locations, types, etc. were recorded. Infrared thermography was used to detect and record transition locations both with and without the trips.

7. The wind tunnel wall geometry had been previously laser scanned for the entire $14 \times 22$ tunnel. Current laser scans of the mast/sting configurations also included tunnel walls in the test section. (The model, including mast and sting in a specific configuration, is shown in Fig. 2.)

8. Laser scans were conducted (wind off) to record the mast and sting configurations (including cabling) in the tunnel at the angles of attack tested with LDV.

9. The angle of attack of the model was monitored throughout the test. Accelerometers on board the model measured acceleration.

10. A few unsteady shear stress sensors were included in the test at selected locations on the model, for evaluation.

11. Unsteady pressures were recorded at several locations on the model.

12. The tunnel's freestream turbulence intensity had been previously measured in the $14 \times 22$ wind tunnel [27]. These measurements were not repeated for this test. The tunnel's freestream turbulence length scale is not known.

13. Triple velocity correlations are possible to obtain from the LDV data taken, although the uncertainty will be significantly higher than for the velocities and second moments. 


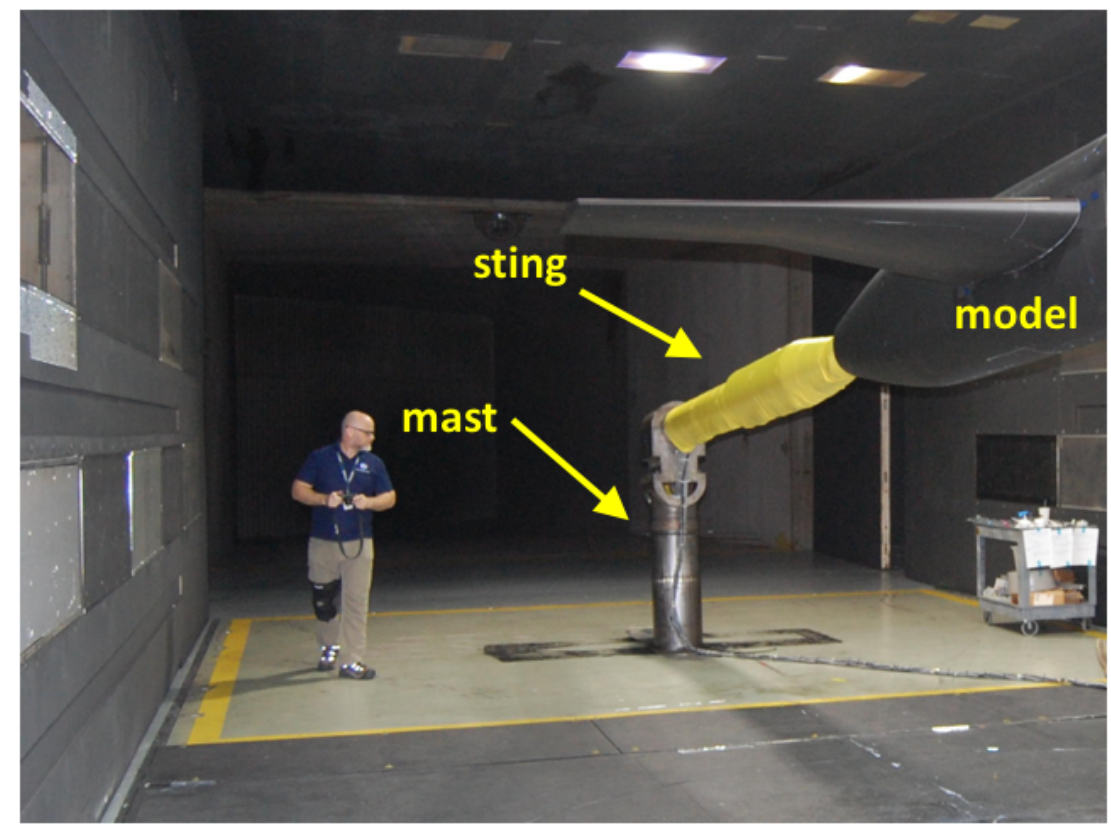

Figure 2. Photograph of JF model in the 14x22 wind tunnel, showing mast and sting.

A complementary experimental accomplishment was made during the exploration of the incoming tunnel freestream as a part of this project: a method for correcting small bias errors (present in the flow angle measurements when using the QWSS measurement device) was devised [11]. This technique allowed useful information to be obtained, and it could prove beneficial for future "freestream" measurements using this or a similarly-designed device.

A few complementary accomplishments have been made on the CFD side as well. Preliminary CFD comparisons were made against the risk-reduction oil flow experiments, and parametric CFD studies including tunnel walls were conducted [12]. And most recently, a CFD automated error controller boundary condition was devised and tested [28]. This latter CFD advance could save a significant amount of time and effort for anyone using CFD to run this type of subsonic wind tunnel case. It also allows for closer matching of the CFD conditions with the wind tunnel conditions.

All of these accomplishments are not only a testament to the skills of the individual JF team members, they also reflect the benefits of team-based planning and organization.

\section{Summary of Lessons Learned}

The Juncture Flow test represents a deliberate and determined effort toward a CFD validation-quality wind tunnel experiment. It is the product of an integrated CFD/experimental team. While there have been challenges, the team has been very productive, and there are several positive takeaways from its experiences.

First and foremost, combining CFD and experimental experts on the same team is very effective. The benefits from the close interactions and different points of view far outweigh any downsides to the collaboration. Also, the more interaction, the more the two sets of experts learn from each other, and the more trust is built. Second, although it is difficult to fully integrate less-active and/or remote members or consultants on a team, including them in regular meetings is still useful because they help bring outside points of view to the table. These points of view increase debate, broaden perspectives, and ultimately improve the end product. And finally, it is important to take time in the beginning of a project to write a Goals Document that summarizes the goals and priorities for the project. Everyone on the team should have input on, and ultimately agree with, this document. Furthermore, it should be reviewed regularly (and modified if necessary) during the course of the project. 


\section{Acknowledgments}

The author acknowledges Bill Oberkampf of WLO Consulting and Joe Morrison and Bil Kleb of NASA Langley for their initial drafting of the JF Goals Document included in the Appendix. The entire JF team (present and past) is also thanked for their hard work and dedication to the project.

\section{Appendix}

\section{Goals for the NASA Juncture Flow Model Validation Experiment}

\section{Introduction}

True validation experiments are designed and executed for the primary purpose of assessing the predictive capability of mathematical models. With this in mind, it is very helpful and constructive to formulate a prioritized list of goals for the NASA Juncture Flow Model (JFM) validation experiment. The formulation of these goals by the project team is not only a constructive process in itself, but the list of goals can then serve as a reference point for project decisions dealing with the test article design, instrumentation, and wind tunnel test conditions.

The JFM validation experiment is designed to be a high quality CFD validation experiment. Specifically, its primary purpose is to provide experimental data for CFD turbulence model validation, and its goals should not be diluted for other purposes. The JFM flow field is not only of practical importance for flight vehicles, but also because it displays competing physical effects that impact turbulence modeling and flow separation. If it is successful, the experimental data will be used by a large number of turbulence modelers and CFD researchers and practitioners.

In setting the goals for the experiment we do not presume any specific type of mathematical model for predicting the JFM flow field. We could reasonably expect that the range of physics modeling fidelity will be:

1. Reynolds-Averaged Navier-Stokes (RANS) models with a likely range from 1-equation through 7-equations for turbulence closure.

2. Hybrid models such as delayed detached eddy simulation (DDES) type models, which represent a blend of RANS near walls and LES in free shear layers and wakes.

3. Large eddy simulation (LES) models.

4. Direct numerical simulation (DNS).

For a given mathematical model of the flow physics and boundary conditions, there are a wide range of methods for the numerical solution of the discretized equations. With adequate code and solution verification, however, each solution method for a given discretized model is expected to converge to the same solution to the mathematical model of the physics. As a result, the goals of the validation experiment do not address any issues related to software correctness, numerical solution methods, or numerical convergence. This document concentrates on the goals of the validation experiment, the experimental measurements that are needed for input to the mathematical models, and the experimental measurements needed to assess the accuracy of the predictions of the models.

With a wide range of possible modeling approaches to the JFM flow field, it is expected that each class of models will yield different levels of predictive capability for various fluid dynamic response quantities. Consequently, a wide range of flow field measurements should be made so as to provide a spectrum of predictive difficulty applicable for the full range of mathematical models. Stated differently, it is desired to measure a wide range of quantities so that all of the mathematical models, including sub-model choices, will have sufficient information for an assessment of their respective predictive capabilities. Using RANS as an example, we would like to be able to not only assess the predictive capabilities of various complete RANS models, but also to assess their component pressure-strain correlation models, diffusion models, etc.

\section{Goals of the Experiment}

Considering the wide range of mathematical models and the experimental instrumentation techniques available, the goal of the JFM validation experiment is to obtain experimental data so that the following prioritized validation assessments of existing models can be made (top priority first):

1. Assess the ability of existing models to predict the onset and extent of the three-dimensionally separated flow near the Wing Juncture Trailing Edge (WJTE) region of a full-span wing-body configuration, in terms of the 
surface topology of the flowfield structure. To provide a range of prediction difficulty, a variation of WJTE flow fields are required, including the onset and progression of corner separation.

2. Assess the ability of the models to predict the time-averaged velocity components, $\mathrm{U}, \mathrm{V}$, and $\mathrm{W}$, at a large number of locations. These locations include several planes of data along the fuselage (upstream of the wing) and multiple planes of data near the wing and body between the leading edge and the trailing edge, with a focus in the WJTE region. These locations include outside and within the boundary layer and the separated flow region, and as close to the walls as possible (at least within a y+ of 100).

3. Assess the ability of the models to predict the components of the turbulent stress tensor, such as Reynolds stresses, at the same locations given in Item 2.

4. Assess the ability of the models to predict the time-averaged surface pressure at a number of surface locations. These include locations on the body and on the wing, with a focus near the WJTE region. Assessments are also desired along chordwise lines out the wing, including near the wing tip; and along spanwise lines, particularly aft of mid-chord.

5. Assess the ability of the models to predict the surface shear stress at a number of locations on the wing and body, particularly near the WJTE region.

6. Assess the ability of temporally-accurate models to predict the power spectral density of measured flow field quantities and surface pressures at a number of locations on the wing and body, including near the WJTE region.

7. Same as Item 3 for triple moments.

To attain this wide range of goals, the experimental data will need to be obtained over a range of wind tunnel conditions. The list of controllable wind tunnel conditions are prioritized as follows (top priority first):

1. Angle of attack (alpha) of the aircraft model.

2. Free stream Reynolds number.

3. Free stream Mach number.

The primary controllable condition that will be used is the angle of attack of the test article.

\section{Input Data and Uncertainties}

To attain the above listed Goals of the Experiment, two features must be designed into the experiment. First, we must carefully measure all of the anticipated input data that various mathematical models may require concerning the experiment. "Input data" to the mathematical model refers to independent information that must be provided to obtain a solution to the partial differential equations. These data can be divided into five general categories: system geometry, initial conditions, system modeling parameters, boundary conditions, and system excitation. For example, we need to carefully characterize the test article geometry and surface features, thermo-physical properties of the air in the wind tunnel, inflow and test section boundary conditions, and, if needed, the acoustic environment in the test section. This information needs to be measured so that it will be easier to isolate the effects of the mathematical model assumptions and approximations, as opposed to the uncertainty associated with unmeasured input data for the mathematical models. Stated differently, we are attempting to greatly reduce the number and importance of adjustable parameters available to the analyst so as to critically assess the key assumptions and approximations of the modeling approach.

Second, we must measure the uncertainties associated with each of the input quantities that are needed by the mathematical models. If the uncertainty of a model input quantity is small, and if the quantity produces little change in the system response quantities of interest, then the analyst will probably choose to consider these inputs as fixed, deterministic, quantities. However, if the uncertainty of an input quantity is large and/or if the uncertainty in the quantity produces large changes in the system response quantities of interest, then the analyst may choose to conduct a non-deterministic simulation.

The following prioritized list of input data for the mathematical models should be obtained and documented for the analysts (top priority first):

1. Documentation of the test article geometry and surface, as fabricated, as assembled, and as tested in the wind tunnel. 
2. Tunnel boundary conditions upstream, around, and downstream of the test article for each alpha condition tested.

3. Mean velocity components through the boundary layer on the fuselage and wing (both upstream and within the WJTE region).

4. Second moments (Reynolds stress tensor components) through the boundary layer (same locations as in Item $3)$.

5. Time-averaged (steady) surface pressure coefficients on the fuselage and wing.

6. If boundary layer trip(s) are used, details regarding their location, type, attachment, etc.

7. Documentation of the surface geometry of the wind tunnel test section and the contraction section upstream of the test section.

8. Documentation of the geometry of the mounting hardware and cabling for the test article in the test section.

9. Time-series measurements of angular position and acceleration on-board the test article.

10. Surface shear stresses on the test article (both upstream and within the WJTE region).

11. Time history and spectra of unsteady velocity and pressure at various locations on the fuselage and wing (both upstream and within the WJTE region).

12. Freestream turbulence intensity and turbulence length scale.

13. Triple velocity correlations through the boundary layer (same locations as in Item 3).

All of the mathematical model input data described above will include a rigorous estimation of total experimental uncertainty, i.e., both random (precision) uncertainty and systematic (bias) uncertainty. The total uncertainty in wind tunnel experiments is commonly divided into three groups of sources; random uncertainty, flow field non-uniformity uncertainty, and test article and instrumentation uncertainty. The contribution from each of these sources can be estimated using statistical design of experiments (DOE) techniques and these are discussed in detail in Refs. [26, 29].

\section{References}

${ }^{1}$ Gand, F., Deck, S., Brunet, V., and Sagaut, P., "Flow Dynamics Past a Simplified Wing Body Junction," Physics of Fluids, Vol. 22, 2010, 115111.

${ }^{2}$ Wood, D. H. and Westphal, R. V., "Measurements of the Flow Around a Lifting-Wing/Body Junction," AIAA Journal, Vol. 30, No. 1, 1992, pp. 6-12.

${ }^{3}$ Barber, T. J., "An Investigation of Strut-Wall Intersection Losses," Journal of Aircraft, Vol. 15, No. 10, 1978, pp. 676-681.

${ }^{4}$ Simpson, R. L., "Junction Flows," Annual Review of Fluid Mechanics, Vol. 33, 2001, pp. 415-443.

${ }^{5}$ Gand, F., Monnier, J.-C., Deluc, J.-M., and Choffat, A., "Experimental Study of the Corner Flow Separation on a Simplified Junction,” AIAA Journal, Vol. 53, No. 10, 2015, pp. 2869-2877.

${ }^{6}$ Bordji, M., Gand, F., Deck, S., and Brunet, V., "Investigation of a Nonlinear Reynolds-Averaged Navier-Stokes Closure for Corner Flows," AIAA Journal, Vol. 54, No. 2, 2016, pp. 386-398, DOI: 10.2514/1.J054313.

${ }^{7}$ Rumsey, C. L., Neuhart, D. H., Kegerise, M. A., “The NASA Juncture Flow Experiment: Goals, Progress, and Preliminary Testing," AIAA Paper 2016-1557, January 2016.

${ }^{8}$ Kegerise, M. A. and Neuhart, D. H., "Wind Tunnel Test of a Risk-Reduction Wing/Fuselage Model to Examine Juncture-Flow Phenomena," NASA TM219348, November 2016

${ }^{9}$ Rumsey, C. L. and Morrison, J. H., "Goals and Status of the NASA Juncture Flow Experiment," NATO Science and Technology Organization, Specialists' Meeting on Progress and Challenges in Validation Testing for Computational Fluid Dynamics, AVT-246-RSM-038, Avila, Spain, 26-28 September 2016, Paper Number AVT-246-03.

${ }^{10}$ Kuester, M. S., Borgoltz, A. and Devenport, W., "Experimental Visualization of Junction Separation Bubbles at Low- to Moderate-Reynolds Numbers," AIAA-2016-3880, June 2016.

${ }^{11}$ Rumsey, C. L., Carlson, J.-R., Hannon, J. A., Jenkins, L. N., Bartram, S. M., Pulliam, T. H., and Lee, H. C., "Boundary Condition Study for the Juncture Flow Experiment in the NASA Langley 14x22-Foot Subsonic Wind Tunnel," AIAA-2017-4126, June 2017.

${ }^{12}$ Lee, H. C., Pulliam, T. H., Neuhart, D. H., and Kegerise, M. A., "CFD Analysis in Advance of the NASA Juncture Flow Experiment," AIAA-2017-4127, June 2017.

${ }^{13}$ Oberkampf, W. L. and Smith, B. L., "Assessment Criteria for Computational Fluid Dynamics Model Validation Experiments," Journal of Verification, Validation and Uncertainty Quantification, Vol. 2, September 2017, pp. 031002-1-031002-14.

${ }^{14}$ Aeschliman, D. P. and Oberkampf, W. L., "Experimental Methodology for Computational Fluid Dynamics Code Validation," AIAA Journal, Vol. 36, No. 5, 1998, pp. 733-741. 
${ }^{15}$ Vassberg, J. C., Tinoco, E. N., Mani, M., Brodersen, O. P., Eisfeld, B., Wahls, R. A., Morrison, J. H., Zickuhr, T., Laflin, K. R., and Mavriplis, D. J., "Abridged Summary of the Third AIAA Computational Fluid Dynamics Drag Prediction Workshop," Journal of Aircraft, Vol. 45, No. 3, 2008, pp. 781-798.

${ }^{16}$ Levy, D., Laflin, K., Tinoco, E., Vassberg, J., Mani, M., Rider, B., Rumsey, C., Wahls, R., Morrison, J., Broderson, O., Crippa, S., Mavriplis, D., and Murayama, M., "Summary of Data from the Fifth Computational Fluid Dynamics Drag Prediction Workshop," Journal of Aircraft, Vol. 51, No. 4, 2014, pp. 1194-1213.

${ }^{17}$ Sclafani, A. J., Vassberg, J. C., Harrison, N. A., Rumsey, C. L., Rivers, S. M., and Morrison, J. H., "CFL3D/OVERFLOW Results for DLR-F6 Wing/Body and Drag Prediction Workshop Wing," Journal of Aircraft, Vol. 45, No. 3, 2008, pp. 762-780.

${ }^{18}$ Eliasson, P., Nordstrom, J., Peng, S.-H., and Tysell, L., "Effect of Edge-based Discretization Schemes in Computations of the DLR F6 Wing-Body Configuration,” AIAA Paper 2008-4153, June 2008.

${ }^{19}$ Sclafani, A. J., Vassberg, J. C., Winkler, C., Dorgan, A. J., Mani, M., Olsen, M. E., and Coder, J. G., "Analysis of the Common Research Model Using Structured and Unstructured Mashes," Journal of Aircraft, Vol. 51, No. 4, 2014, pp. 1223-1243.

${ }^{20}$ Yamamoto, K., Tanaka, K., and Murayama, M., "Comparison Study of Drag Prediction for the 4th CFD Drag Prediction Workshop using Structured and Unstructured Mesh Methods," AIAA Paper 2010-4222, June-July 2010.

${ }^{21}$ Spalart, P. R., "Strategies for Turbulence Modelling and Simulation," International Journal of Heat and Fluid Flow, Vol. 21, 2000, pp. 252-263.

${ }^{22}$ Dandois, J., "Improvement of Corner Flow Prediction Using the Quadratic Constitutive Relation," AIAA Journal, Vol. 52, No. 12, 2014, pp. $2795-2806$.

${ }^{23}$ Rumsey, C. L., "Application of Reynolds Stress Models to Separated Aerodynamic Flows," in Differential Reynolds Stress Modeling for Separating Flows in Industrial Aerodynamics, ed: B. Eisfeld, Springer Tracts in Mechanical Engineering, Springer International Publishing, Cham, Switzerland, 2015, pp. 19-37.

${ }^{24}$ Kusunose, K., "Lift Analysis Based on a Wake-Integral Method," AIAA Paper 2001-0420, January 2001.

${ }^{25}$ Kusunose, K. and Crowder, J. P., "Extension of Wake-Survey Analysis Method to Cover Compressible Flows," Journal of Aircraft, Vol. 39 , No. 6, 2002, pp. 954-963.

${ }^{26}$ Rhode, M. N. and Oberkampf, W. L., "Estimation of Uncertainties for a Supersonic Retro-Propulsion Model Validation Experiment in a Wind Tunnel", AIAA Paper 2012-2707, June 2012.

${ }^{27}$ Neuhart, D. H. and McGinley, C. B., "Free-Stream Turbulence Intensity in the Langley 14- by 22-Foot Subsonic Tunnel," NASA/TP-2004213247, August 2004.

${ }^{28}$ Carlson, J.-R., “Automated Boundary Conditions for Wind Tunnel Simulations,” NASA/TM-2018-219812, March 2018.

${ }^{29}$ Oberkampf, W. L. and Roy, C. J., Verification and Validation in Scientific Computing, Cambridge, UK: Cambridge University Press, 2010. 